\title{
Toeplitz operators with radial symbols on weighted holomorphic Orlicz space
}

\section{Karapetyants, Alexey}

Birkhäuser

2020

Karapetyants , A \& Taskinen , J 2020 , Toeplitz operators with radial symbols on weighted holomorphic Orlicz space . in W Bauer, R Duduchava, S Grudsky \& M Kaashoek (eds), Operator algebras, Toeplitz operators and related topics . Operator Theory: Advances and Applications , vol. 279 , Birkhäuser , pp. 189-204 . https://doi.org/10.1007/978-3-030-44651-2_14

http://hdl.handle.net/10138/340049

https://doi.org/10.1007/978-3-030-44651-2_14

acceptedVersion

Downloaded from Helda, University of Helsinki institutional repository.

This is an electronic reprint of the original article.

This reprint may differ from the original in pagination and typographic detail.

Please cite the original version. 


\title{
Toeplitz operators with radial symbols on weighted holomorphic Orlicz space
}

\author{
Alexey Karapetyants (Southern Federal University, Russia, karapetyants@ gmail.com) \\ and \\ Jari Taskinen (University of Helsinki, Finland, jari.taskinen@ @elsinki.fi ) \\ Dedicated to the occasion of 70th birthday of Professor N.Vasilevski.
}

\begin{abstract}
We consider a class of Toeplitz operators with special radial symbols on weighted holomorphic Orlicz space. For an operator from such class we prove necessary and sufficient conditions of boundedness on holomorphic Orlicz space in terms of behaviour of averages of the radial symbol of the operator. In the case when either symbol or its certain average is nonnegative, we obtain characterization for boundedness of the corresponding Toeplitz operator.
\end{abstract}

\section{Introduction}

The weighted holomorphic Orlicz space $\mathcal{A}_{\lambda}^{\Phi}(\mathbb{D})$ on the unit disc $\mathbb{D}$ in the complex plane $\mathbb{C}$ is defined to consists of the functions from the weighted Orlicz space $L_{\lambda}^{\Phi}(\mathbb{D})$ which are also holomorphics in $\mathbb{D}$ (for details see Section 2). This is a direct generalization of the weighted classical Bergman space, sometimes called Bergman-Jerbashian space. We refer to the books [8, 4, 24, 27, 28] for a general modern theory of Bergman type spaces and operators on Bergman type spaces. For the definition of Orlicz space and some properties we refer to the corresponding section in this paper and also to the books $[9,13,19,18,14]$.

Recently, the study of classical operators of complex analysis, such as Toeplitz, Hankel and composition operators, has attracted considerable attention in the framework of the holomorphic Orlicz spaces and their modification, see e.g. [2, $1,12,23,11,10]$.

Toeplitz operators form an important and much studied subclass of the classical operators. Given a symbol $a \in L_{\lambda}^{1}(\mathbb{D})$ on the unit disc and $\lambda>-1$, the Toeplitz operator $T_{a}^{\lambda}$ is defined by the formula

$$
T_{a}^{(\lambda)} f=P_{\lambda}(a f),
$$


where

$$
P_{\lambda} f(z)=\int_{\mathbb{D}} \frac{f(w)}{(1-z \bar{w})^{2+\lambda}} d A_{\lambda}(w), \quad z \in \mathbb{D},
$$

is the classical Bergman projection; here, $d A_{\lambda}=\left(1-|z|^{2}\right)^{\lambda} d A$ and $d A$ is the area measure on the complex plane normalized so that the measure of the disc equals 1 . For an arbitrary $a \in L_{\lambda}^{1}(\mathbb{D})$ the operator $T_{a}^{(\lambda)}$ may fail to map the standard weighted Bergman space $\mathcal{A}_{\lambda}^{p}(\mathbb{D})$ into itself but anyway it is densely defined, namely on the subspace $H^{\infty}(\mathbb{D})$ of bounded analytic functions.

The question of characterizing symbols $a \in L_{\lambda}^{1}(\mathbb{D})$ such that the corresponding Toeplitz operator is bounded in $\mathcal{A}_{\lambda}^{p}(\mathbb{D})$ is still open even in the simplest case $p=2, \lambda=0$. There are however special cases, when a satisfactory or even complete answer is known. Bounded Toeplitz operators with positive symbols on $\mathcal{A}^{2}(\mathbb{D})$ and more general spaces were characterized in [17] and [26]. An extensive study of Toelpitz operators with (unbounded) radial symbols can be found in the [5, 6, 7], see also the monograph by Vasilevski, [24]. Zorboska [29] considered $L^{1}(\mathbb{D})$-symbols that satisfy the condition of bounded mean oscillation and determined the bounded Toeplitz operators in terms of the boundary behavior of the Berezin transform of their symbols.

The purpose of this work is to extend to weighted Orlicz space $\mathcal{A}_{\lambda}^{\Phi}(\mathbb{D})$ setting certain results concerning the boundedness of $T_{a}^{(\lambda)}$ for radial symbols. It is well known (see Theorem 7.5 in [28]), that the boundedness can be characterized in the case of standard weighted Bergman spaces and positive symbols. In [15], generalizing the results in [5], [24], the positivity requirement was considerably relaxed by a much weaker assumption on the positivity of certain repeated integrals of the (radial) symbol. The proof used certain estimates of the kernel of the Berezin transform. Here, we will give a further generalization of this argument to the case of Orlicz spaces, see Theorems 3.3, 3.4 and Theorem 3.5, where the result is formulated as a characterization of the boundedness of the Toeplitz operator and its Berezin transform.

The paper is organized as follows. In Section 2 we give some necessary definitions and collect auxiliary results. Section 3 is devoted to our main results. In Theorem 3.3 we give sufficient conditions for boundedness of Toeplitz operator with radial symbol in holomorphic Orlicz space in terms of behaviour of averages of the symbol under some additional conditions on Young function, which, roughly speaking, allow power-like Young functions. We also notice that in that case the Berezin transform of the Toeplitz operator is a bounded function. Without these power-like additional conditions for Young function, but under condition 
of positivity either of a symbol or a certain average of the symbol we prove the necessity: i.e., boundedness of Toeplitz operator and finiteness of Berezin transform imply certain behavior of average, see Theorem 3.4. In Theorem 3.5 we collect the above information in one statement, so presenting a characterization for boundedness of Toeplitz operators with radial symbols and with a condition that either symbol or certain average of this operator is a nonnegative function. We also present a reformulation for the weighted Lebesgue case, i.e. when the Young function is given by $\Phi(t)=t^{p}$.

Acknowledgements Alexey Karapetyants is partially supported by the Russian Foundation for Fundamental Research, projects 18-01-00094 and 18-51-05009.

\section{Preliminaries}

\subsection{Notations}

Given $1 \leq p \leq \infty$ and $-1<\lambda<\infty$ we denote by $L_{\lambda}^{p}(\mathbb{D})$ the standard Lebesgue space of complex valued functions on the disc $\mathbb{D}$ that are $p$ - integrable with respect to the measure $d A_{\lambda}$. By $\mathcal{A}_{\lambda}^{p}(\mathbb{D})$ we denote the standard weighted Bergman space, which is the closed subspace of $L_{\lambda}^{p}(\mathbb{D})$ consisting of analytic functions in $\mathbb{D}$. The index $\lambda$ is suppressed from the notation, if it equals 0 . By $C_{0}^{\infty}(\mathbb{D})$ we denote the space of compactly supported $C^{\infty}$-functions on the disc. Given an analytic function $f$ and $m \in \mathbb{N}=\{1,2,3, \ldots\}$ we write $f^{(m)}$ for the $m$-th complex derivative of $f$. By $C, C_{1}$ etc. we denote generic positive constant(s), the value of which may change from place to place but not in the same group of inequalities. We use the Landau notation so that for example " $f(r)=O(g(r)), r \rightarrow 1$ " means that the quantity $f(r) / g(r)$ remains bounded in the limit $r \rightarrow 1$.

\subsection{Orlicz spaces}

Let us recall the definition of the weighted Orlicz space $L_{\lambda}^{\Phi}(\mathbb{D})$ and some properties of Young functions $\Phi$. For more details we refer the reader to the books $[9,13,19,18,14]$. Let $\Phi:[0, \infty] \rightarrow[0, \infty]$ be a Young function, i.e., a convex function such that $\Phi(0)=0, \lim _{x \rightarrow \infty} \Phi(x)=\Phi(\infty)=\infty$. From the convexity and $\Phi(0)=0$ it follows that any Young function is increasing. To each Young function $\Phi$ one identifies the complementary function $\Psi$, which possesses 
the same properties, by the rule $\Psi(y)=\sup _{x \geqslant 0}\{x y-\Phi(x)\}$. Note that

$$
t \leqslant \Phi^{-1}(t) \Psi^{-1}(t) \leqslant 2 t \text { for all } t \geqslant 0
$$

Let $L_{\lambda}^{\Phi}(\mathbb{D})$ be the weighted Orlicz space consisting of all measurable functions $f$ on $\mathbb{D}$ such that

$$
\int_{\mathbb{D}} \Phi(k|f(z)|) d A_{\lambda}(z)<\infty, \text { for some } k>0 .
$$

The functional

$$
N_{\Phi}(f)=\|f\|_{L_{\lambda}^{\Phi}(\mathbb{D})}=\inf \left\{\lambda>0: \int_{\mathbb{D}} \Phi\left(\frac{|f(z)|}{\lambda}\right) d A_{\lambda}(z) \leqslant 1\right\}
$$

defines a norm in $L_{\lambda}^{\Phi}(\mathbb{D})$. By definition, $L_{\lambda}^{\Phi}(\mathbb{D})$ is a lattice, i.e.

$$
\begin{aligned}
& f \in L_{\lambda}^{\Phi}(\mathbb{D}) \text { and }|g(z)| \leq|f(z)| \text { for a.e. } z \in \mathbb{D} \Rightarrow \\
& g \in L_{\lambda}^{\Phi}(\mathbb{D}) \text { and }\|g\|_{L_{\lambda}^{\Phi}(\mathbb{D})} \leq\|f\|_{L_{\lambda}^{\Phi}(\mathbb{D})} .
\end{aligned}
$$

We will need the following indices

$$
\begin{aligned}
& p_{\Phi}=\sup \left\{s>0: t^{-s} \Phi(t) \text { is non }- \text { decreasing for } \mathrm{t}>0\right\} \\
& q_{\Phi}=\inf \left\{s>0: t^{-s} \Phi(t) \text { is non }- \text { increasing for } \mathrm{t}>0\right\} .
\end{aligned}
$$

These indices were used first by Yamamuro [25] (see also [16]).

The following density result is a reformulation of Theorem 3.7.15 of [9], where the unweighted version is presented, and it can be proved by the same arguments as in the reference.

Lemma 2.1. Let $\Phi$ be a Young function and there exists $q<\infty$ such that $\frac{\Phi(t)}{t^{q}}$ is almost decreasing. Then the space $C_{0}^{\infty}(\mathbb{D})$ is a dense subspace of $L_{\lambda}^{\Phi}(\mathbb{D})$.

Proof. Our space $L_{\lambda}^{\Phi}(\mathbb{D})$ equals the space $L^{\varphi}(A, d \mu)$ in Proposition 3.5.1 of [9], when $\mathbb{D}, \Phi$ and $d A_{\lambda}$ are taken as $A, \varphi$ and $d \mu$, respectively. Thus, according to the citation, simple functions (see e.g. Section 3.5. of [9]) on the unit disc form a dense subspace $L_{\lambda}^{\Phi}(\mathbb{D})$. Furthermore, by classical, elementary arguments, simple functions can be approximated by functions of $C_{0}^{\infty}(\mathbb{D})$ simultaneously with respect to the norms of $L_{\lambda}^{p}(\mathbb{D})$ and $L_{\lambda}^{q}(\mathbb{D})$. Now, according to Lemma 3.7.7 of [9], the space $L_{\lambda}^{p}(\mathbb{D}) \cap L_{\lambda}^{q}(\mathbb{D})$ embeds continuously into $L_{\lambda}^{\Phi}(\mathbb{D})$. This yields the result. 


\subsection{Classical operators}

Along with the weighted Bergman projection $P_{\lambda}$, we will use the following operators, which are modifications of the classical Bergman projections:

$$
\begin{aligned}
P_{\lambda}^{+} f(z) & =\int_{\mathbb{D}} \frac{f(w)}{|1-z \bar{w}|^{2+\lambda}} d A_{\lambda}(w), \\
T_{a, b} f(z) & =\left(1-|z|^{2}\right)^{a} \int_{\mathbb{D}} \frac{f(w)}{(1-z \bar{w})^{2+a+b}} d A_{b}(w), \\
S_{a, b} f(z) & =\left(1-|z|^{2}\right)^{a} \int_{\mathbb{D}} \frac{f(w)}{|1-z \bar{w}|^{2+a+b}} d A_{b}(w) .
\end{aligned}
$$

Theorem 2.2. ([3],[20]). Let $\lambda, \beta>-1$. Let $\Phi$ be a Young function and assume that $1<p_{\Phi} \leqslant q_{\Phi}<\infty$ and $\lambda+1<p_{\Phi}(\beta+1)$. Then

1. $P_{\beta}$ is bounded in $L_{\lambda}^{\Phi}(\mathbb{D})$;

2. $P_{\beta}^{+}$is bounded in $L_{\lambda}^{\Phi}(\mathbb{D})$.

The Theorem 2.2 was proved in [3] (and generalized to the weighted setting in [20]) for the case of the Bergman projection and under some slightly different assumption for Young function. In the citations the authors use the so-called lower and upper indices, introduced and used first by Simonenko [21] in the context of interpolation and extrapolation of Orlicz spaces. Here we use different Yamamuro type indices $p_{\Phi}, q_{\Phi}$, and we do not assume continuity of Young function. But, the proof of this modified version is the same as the proof in [20], moreover it clearly holds for the maximal Bergman operator.

We also need some slight generalization of the above result.

Theorem 2.3. Let $\Phi$ be a Young function and assume that $1<p_{\Phi} \leqslant q_{\Phi}<\infty$. Let $a \geqslant 0, \lambda>-1, b>-1$, and $\lambda+1<p_{\Phi}(\beta+1)$. Then

1. $T_{a, b}$ is bounded in $L_{\lambda}^{\Phi}(\mathbb{D})$;

2. $S_{a, b}$ is bounded in $L_{\lambda}^{\Phi}(\mathbb{D})$.

Proof. There exists $p_{0}, 1<p_{0}<p_{\Phi}$, such that $-p_{0} a<\lambda+1<p_{0}(b+1)$. Certainly, there exists $p_{1}>q_{\Phi}$ such that $-p_{1} a<\lambda+1<p_{1}(b+1)$. Therefore by Theorem 2.10 from [27] the operators $T_{a, b}$ and $S_{a, b}$ are bounded on $L_{\lambda}^{p_{0}}(\mathbb{D})$ and on $L_{\lambda}^{p_{1}}(\mathbb{D})$. The rest of the proof follows by interpolation using Theorem 6.5 , part (c) from [16]. 
Theorem 2.4. Let $\Phi$ be a Young function and assume that $1<p_{\Phi} \leqslant q_{\Phi}<\infty$. Let $\lambda>-1, m \in \mathbb{N}$ and let $f$ be an analytic function on $\mathbb{D}$ and denote

$$
g_{m}(z)=\sum_{k=0}^{m}\left(1-|z|^{2}\right)^{k} f^{(k)}(z)
$$

If $f \in \mathcal{A}_{\lambda}^{\Phi}(\mathbb{D})$, then $g_{m}$ belongs to $\mathcal{A}_{\lambda}^{\Phi}(\mathbb{D})$ and there holds the norm bound

$$
\left\|g_{m}\right\|_{L_{\lambda}^{\Phi}(\mathbb{D})} \leqslant C_{\Phi, \lambda, m}\|f\|_{L_{\lambda}^{\Phi}(\mathbb{D})}
$$

for some positive constant $C_{\Phi, \lambda, m}$ depending on $\Phi, \lambda$, and $m$.

Proof. Assume first $f \in \mathcal{A}_{\lambda}^{\Phi}(\mathbb{D})$. We choose $b>0$ so large that $\lambda+1<p_{\Phi}(b+1)$ and differentiate the reproducing formula

$$
f(z)=C_{b} \int_{\mathbb{D}} \frac{\left(1-|w|^{2}\right)^{b}}{(1-z \bar{w})^{2+b}} f(w) d A(w)
$$

with respect to $z$ under the integral sign several times to write for all $k=1, \ldots, m$

$$
\left(1-|z|^{2}\right)^{k} f^{(k)}(z)=C_{k, b}\left(1-|z|^{2}\right)^{k} \int_{\mathbb{D}} \frac{\left(1-|w|^{2}\right)^{b}}{(1-z \bar{w})^{2+k+b}} \bar{w}^{k} f(w) d A(w),
$$

Here, the function $\bar{z}^{k} f$ belongs $L_{\lambda}^{\Phi}(\mathbb{D})$, by (2.2), hence the right hand side also does by Theorem 2.3, since it is equal to $T_{k, b}\left(\bar{z}^{k} f\right)$. The norm bound (2.6) is clear due to (2.2).

\subsection{Berezin transform}

Recall that, on the unit disc, the weighted Berezin transform of a function $f$ is defined by

$$
\mathbb{B}_{\lambda} f(z) \equiv \tilde{f}_{\lambda}(z)=\int_{\mathbb{D}} f\left(\varphi_{z}(w)\right) d A_{\lambda}(w)=\int_{\mathbb{D}} f(w)\left|k_{z}^{\lambda}(w)\right|^{2} d A_{\lambda}(w) .
$$

Here

$$
k_{z}^{\lambda}(w)=\frac{\left(1-|z|^{2}\right)^{1+\lambda / 2}}{(1-z \bar{w})^{2+\lambda}}
$$

are the normalized weighted reproducing kernels of the classical weighted Bergman space $\mathcal{A}_{\lambda}^{2}(\mathbb{D})$ and $\varphi_{z}(w)=(z-w) /(1-\bar{z} w)$ is the Moebius transform of the unit 
disc. The (weighted) Berezin transform of a bounded operator $T: \mathcal{A}_{\lambda}^{2}(\mathbb{D}) \rightarrow$ $\mathcal{A}_{\lambda}^{2}(\mathbb{D})$ is defined as

$$
\widetilde{T}(z)=\left\langle T k_{z}^{\lambda}, k_{z}^{\lambda}\right\rangle_{\lambda}=\int_{\mathbb{D}} T k_{z}^{\lambda}(w) \overline{k_{z}^{\lambda}(w)} d A_{\lambda}(w) .
$$

The Berezin transform $\widetilde{T}_{a}^{(\lambda)}$ of the bounded Toeplitz operator $T_{a}^{(\lambda)}$ in $\mathcal{A}_{\lambda}^{2}(\mathbb{D})$ with symbol $a$ coincides with the Berezin transform of the symbol $\widetilde{a}_{\lambda}$. See $[8,27,28]$ for details.

If $-1<\lambda<\infty$ and $X$ is any Banach space of analytic functions on the disc such that all bounded analytic functions are contained both to $X$ and its dual $X^{*}$ (determined by the integral duality with respect to the measure $d A_{\lambda}$ ), and $T: X \rightarrow X$ is a bounded operator, we define the Berezin transform $\widetilde{T}$ of $T$ by the same formula (2.9).

Remark 2.5. It is known in the case of standard Bergman spaces that if the Toeplitz operator $T_{a}^{(\lambda)}$ maps $\mathcal{A}_{\lambda}^{p}(\mathbb{D})$ boundedly into itself, then its Berezin transform is a bounded function on the disc $\mathbb{D}$. In fact, this follows directly from the boundedness of $T_{a}^{(\lambda)}$ and the definition of $\widetilde{T}_{a}^{(\lambda)}$, since the norm of $k_{z}^{\lambda}$ in $\mathcal{A}_{\lambda}^{p}(\mathbb{D})$, respectively in $\mathcal{A}_{\lambda}^{q}(\mathbb{D})$ with $1 / p+1 / q=1$, is proportional to $\left(1-|z|^{2}\right)^{-1-\frac{\lambda}{2}+\frac{\lambda+2}{p}}$, respectively $\left(1-|z|^{2}\right)^{-1-\frac{\lambda}{2}+\frac{\lambda+2}{q}}$; see also Theorem 7.5 of [28] and paper [22]. We do not known in what generality this result extends to Orlicz spaces.

\section{Toeplitz operators with radial symbols on $\mathcal{A}_{\lambda}^{\Phi}(\mathbb{D})$}

We start by an elementary technical statement, but we prefer to prove it explicitly in order to show the dependence of the estimates on the constants.

Lemma 3.1. For $w \in \mathbb{D}$, denote $w=\rho \sigma$, where $\rho=|w|$ and $\sigma=\frac{w}{|w|}$. There exist a constant $C$ such that

$$
\frac{1}{C(\gamma-1)} \frac{1}{(1-\rho|z|)^{\gamma-1}} \leqslant \int_{\mathbb{T}} \frac{|d \sigma|}{|1-z \bar{w}|^{\gamma}} \leqslant \frac{C}{\gamma-1} \frac{1}{(1-\rho|z|)^{\gamma-1}}
$$

Proof. If $r=|z| \leqslant \frac{1}{2}$ the two sided estimate (3.1) is obvious even with only constants in the left and right side.

Let $r \geqslant \frac{1}{2}$. We have with the notation $\delta=1-\rho|z|$ :

$$
I_{\gamma}(z, \rho)=\int_{\mathbb{T}} \frac{|d \sigma|}{|1-z \bar{w}|^{\gamma}}=\int_{\mathbb{T}} \frac{|d \sigma|}{|\sigma-z \rho|^{\gamma}}=4 \int_{0}^{\frac{\pi}{2}} \frac{d \alpha}{\left(\delta^{2}+4 r \sin ^{2} \alpha\right)^{\frac{\gamma}{2}}} .
$$


For the estimate from below we note that $\sin \alpha \leqslant \alpha$ on $\left[0, \frac{\pi}{2}\right]$, hence

$$
\delta^{2}+4 r \sin ^{2} \alpha \leqslant \delta^{2}+4 r \alpha^{2} \leqslant(\delta+2 \sqrt{r} \alpha)^{2},
$$

and

$$
\begin{aligned}
I_{\gamma}(z, \rho) & \geqslant 4 \int_{0}^{\frac{\pi}{2}} \frac{d \alpha}{(\delta+2 \sqrt{r} \alpha)^{\gamma}}=\frac{1}{\delta^{\gamma-1}} \frac{2}{\sqrt{r}(\gamma-1)}\left(1-\left(\frac{\delta}{\delta+\pi \sqrt{r}}\right)^{\gamma-1}\right) \\
& \geqslant \frac{1}{\delta^{\gamma-1}} \frac{2}{(\gamma-1)}\left(1-\left(\frac{\delta}{\delta+\frac{\pi}{\sqrt{2}}}\right)^{\gamma-1}\right) \geqslant \frac{C_{1}}{\gamma-1} \frac{1}{(1-\rho|z|)^{\gamma-1}} .
\end{aligned}
$$

For the estimate from above we note that since $\frac{\sin \alpha}{\alpha}$ is decreasing on $\left[0, \frac{\pi}{2}\right]$ one has $\sin \alpha \geqslant \frac{2}{\pi} \alpha$ on $\left[0, \frac{\pi}{2}\right]$, and then we obtain

$$
\delta^{2}+4 r \sin ^{2} \alpha \geqslant \delta^{2}+\frac{16}{\pi^{2}} r \alpha^{2} \geqslant \frac{\left(\delta+\frac{4}{\pi} \sqrt{r} \alpha\right)^{2}}{2} .
$$

Hence,

$$
\begin{aligned}
I_{\gamma}(z, \rho) & \leqslant 2^{2+\frac{\gamma}{2}} \int_{0}^{\frac{\pi}{2}} \frac{d \alpha}{\left(\delta+\frac{4}{\pi} \sqrt{\gamma} \alpha\right)^{\frac{\gamma}{2}}} \leqslant \frac{2^{\frac{\gamma+1}{2}} \pi}{(\gamma-1)} \frac{1}{\delta^{\gamma-1}}\left(1-\left(\frac{\delta}{\delta+2}\right)^{\gamma-1}\right) \\
& \leqslant \frac{C_{2}}{\gamma-1} \frac{1}{(1-\rho|z|)^{\gamma-1}} .
\end{aligned}
$$

Remark 3.2. It is a matter of calculus to show that

$$
\frac{C_{1}}{(\gamma-1)} \frac{1}{(1-\rho|z|)^{\gamma+m-1}} \leqslant \frac{\partial^{m}}{\partial \rho^{m}} \int_{\mathbb{T}} \frac{1}{|1-z \rho \bar{\sigma}|^{\gamma}}|d \sigma| \leqslant \frac{C_{2}}{(\gamma-1)} \frac{1}{(1-\rho|z|)^{\gamma+m-1}},
$$

for $m=0,1,2, \ldots$

We also make a remark that will be needed later: for all $n \in \mathbb{N}$, and $\lambda>-1$ there exists a constant $C_{\Phi, n, \lambda}>0$ such that

$$
\left|f^{(n)}(0)\right| \leq C_{\Phi, n, \lambda}\|f\|_{L_{\lambda}^{\Phi}(\mathbb{D})},
$$

for all $f \in \mathcal{A}_{\lambda}^{\Phi}(\mathbb{D})$. Indeed, for every $0<r<1$ one has by the Cauchy integral formula

$$
f^{(n)}(0)=\frac{n !}{2 \pi i} \int_{0}^{2 \pi} \frac{f\left(r e^{i t}\right)}{r^{n+1} e^{i n t}} r e^{i t} i d t
$$


which yields for example for all $1 / 4<r<3 / 4$

$$
\left|f^{(n)}(0)\right| \leqslant \frac{n !}{2 \pi} \int_{0}^{2 \pi} \frac{\left|f\left(r e^{i t}\right)\right|}{r^{n}} d t \leqslant C \int_{0}^{2 \pi}\left|f\left(r e^{i t}\right)\right|\left(1-r^{2}\right)^{\lambda} r d t
$$

for some constant $C>0$ depending on $n$ and $\lambda$. Integrating both sides over $\left(\frac{1}{4}, \frac{3}{4}\right)$ with respect to $r$, we obtain

$$
\begin{aligned}
\left|f^{(n)}(0)\right| & \leqslant 2 C \int_{\frac{1}{4}}^{\frac{3}{4}} d r \int_{0}^{2 \pi}\left|f\left(r e^{i t}\right)\right|\left(1-r^{2}\right)^{\lambda} r d t \\
& \leqslant C_{1} \int_{\mathbb{D}}|f(z)| d A_{\lambda}(z) \leqslant C_{1}\|f\|_{L_{\lambda}^{\Phi}(\mathbb{D})} .
\end{aligned}
$$

Here we used the Hölder inequality and the fact that the weight $\left(1-r^{2}\right)^{\lambda}$ is bounded from below and above by positive constants for the given $r$-interval.

Now we are in position to formulate and prove our main results. Let us introduce the averages:

$$
\begin{aligned}
B_{a, \lambda}^{(0)}(r) & =a(r) \\
B_{a, \lambda}^{(1)}(r) & =\int_{r}^{1} a(t)\left(1-t^{2}\right)^{\lambda} t d t \\
B_{a, \lambda}^{(j)}(r) & =\int_{r}^{1} B_{a, \lambda}^{(j-1)}(t) d t, \quad j=2,3, \ldots
\end{aligned}
$$

Theorem 3.3. Let $\Phi$ be a Young function with $1<p_{\Phi} \leqslant q_{\Phi}<\infty$. If there exists $m \in \mathbb{N} \cup\{0\}$ such that

(i) $B_{a, \lambda}^{(m)}(r)=O\left((1-r)^{m+\lambda}\right), \quad r \rightarrow 1$,

then

(ii) the Toeplitz operator $T_{a}^{(\lambda)}: \mathcal{A}_{\lambda}^{\Phi}(\mathbb{D}) \rightarrow \mathcal{A}_{\lambda}^{\Phi}(\mathbb{D})$ is well-defined and bounded, and its Berezin transform $\widetilde{T}_{\lambda}$ is a bounded function.

Proof. We first prove the boundedness of the Toeplitz operator. By Lemma 2.1 we know that polynomials form a dense set in $\mathcal{A}_{\lambda}^{\Phi}(\mathbb{D})$, hence at least for $f$ being a polynomial we have:

$$
\begin{aligned}
T_{a}^{(\lambda)} f(z) & =\int_{\mathbb{D}} \frac{a(w) f(w)}{(1-z \bar{w})^{2+\lambda}} d A_{\lambda}(w) \\
& =\frac{\lambda+1}{\pi} \int_{\mathbb{T}}|d \sigma| \int_{0}^{1} \frac{a(\rho) f(\rho \sigma)}{(1-z \rho \bar{\sigma})^{2+\lambda}}\left(1-\rho^{2}\right)^{\lambda} \rho d \rho
\end{aligned}
$$




$$
\begin{aligned}
& =\frac{\lambda+1}{\pi} \int_{\mathbb{T}}\left[\left.\sum_{j=1}^{m} B_{a, \lambda}^{(j)}(0)\left(\frac{\partial^{j-1}}{\partial \rho^{j-1}} \frac{f(\rho \sigma)}{(1-z \rho \bar{\sigma})^{2+\lambda}}\right)\right|_{\rho=0}\right. \\
& \left.+\int_{0}^{1} B_{a, \lambda}^{(m)}(\rho)\left(\frac{\partial^{m}}{\partial \rho^{m}} \frac{f(\rho \sigma)}{(1-z \rho \bar{\sigma})^{2+\lambda}}\right) d \rho\right]|d \sigma| .
\end{aligned}
$$

We note that

$$
\begin{aligned}
& \frac{\partial^{j}}{\partial \rho^{j}} \frac{f(\rho \sigma)}{(1-z \rho \bar{\sigma})^{2+\lambda}}=\sum_{k=0}^{j} C_{k}^{j}\left(\frac{\partial^{k}}{\partial \rho^{k}} f(\rho \sigma)\right)\left(\frac{\partial^{j-k}}{\partial \rho^{j-k}} \frac{1}{(1-z \rho \bar{\sigma})^{2+\lambda}}\right) \\
= & \sum_{k=0}^{j} A_{k, j, \lambda}(z, \sigma) f^{(k)}(\rho \sigma) \frac{1}{(1-z \rho \bar{\sigma})^{2+\lambda+j-k}},
\end{aligned}
$$

where $A_{k, j, \lambda}(z, \sigma)=C_{k, j, \lambda} z^{j-k} \sigma^{2 k-j}$ with some constants $C_{k, j, \lambda}$. Therefore,

$$
\begin{aligned}
T_{a}^{(\lambda)} f(z) & =\frac{\lambda+1}{\pi} \sum_{j=1}^{m} B_{a, \lambda}^{(j)}(0) \sum_{k=0}^{j-1} f^{(k)}(0) \int_{\mathbb{T}} A_{k, j-1, \lambda}(z, \sigma)|d \sigma| \\
& +\frac{\lambda+1}{\pi} \int_{\mathbb{T}}|d \sigma| \int_{0}^{1} B_{a, \lambda}^{(m)}(\rho)\left(\frac{\partial^{m}}{\partial \rho^{m}} \frac{f(\rho \sigma)}{(1-z \rho \bar{\sigma})^{2+\lambda}}\right) d \rho
\end{aligned}
$$

and the first double sum is nonzero only if $j$ is even and $k=\frac{j}{2}$.

Regarding the second term, taking into account that

$$
\frac{\left(1-\rho^{2}\right)^{m-k}}{|1-z \rho \bar{\sigma}|^{2+\lambda+m-k}} \leqslant C \frac{1}{|1-z \rho \bar{\sigma}|^{2+\lambda}}
$$

uniformly in $z \in \mathbb{D}, \sigma \in \mathbb{T}$, we calculate

$$
\begin{aligned}
\left|I_{m, a, \lambda} f(z)\right| & \equiv\left|\int_{\mathbb{T}}\right| d \sigma\left|\int_{0}^{1} B_{a, \lambda}^{(m)}(\rho)\left(\frac{\partial^{m}}{\partial \rho^{m}} \frac{f(\rho \sigma)}{(1-z \rho \bar{\sigma})^{2+\lambda}}\right) d \rho\right| \\
& \leqslant C \int_{\mathbb{T}}|d \sigma| \int_{0}^{1}\left(\sum_{k=0}^{m}\left(1-\rho^{2}\right)^{k}\left|f^{(k)}(\rho \sigma)\right|\right) \frac{\left(1-\rho^{2}\right)^{\lambda}}{|1-z \rho \bar{\sigma}|^{2+\lambda}} d \rho \\
& =C_{1} \int_{\mathbb{D}} \frac{\sum_{k=0}^{m}\left(1-|w|^{2}\right)^{k}\left|f^{(k)}(w)\right|}{|1-z \bar{w}|^{2+\lambda}} d A_{\lambda}(w) \\
& =C_{1} P_{\lambda}^{+} g_{m}(z)
\end{aligned}
$$


where $P_{\lambda}^{+}$is as in (2.3) and we denoted

$$
g_{m}(z)=\sum_{k=0}^{m}\left(1-|z|^{2}\right)^{k} f^{(k)}(z) .
$$

The function $g_{m}$ belongs to $L_{\lambda}^{\Phi}(\mathbb{D})$ due to $f \in \mathcal{A}_{\lambda}^{\Phi}(\mathbb{D})$ and Theorem 2.4, and we have the bound

$$
\left\|g_{m}\right\|_{\mathcal{A}_{\lambda}^{\Phi}(\mathbb{D})} \leqslant C\|f\|_{\mathcal{A}_{\lambda}^{\Phi}(\mathbb{D})}, \quad 1<p<\infty, \lambda>-1 .
$$

This, together with Theorem 2.2 imply $P_{\lambda}^{+} g_{m} \in \mathcal{A}_{\lambda}^{\Phi}(\mathbb{D})$ and

$$
\left\|P_{\lambda}^{+} g_{m}\right\|_{\mathcal{A}_{\lambda}^{\Phi}(\mathbb{D})} \leqslant C\left\|g_{m}\right\|_{\mathcal{A}_{\lambda}^{\Phi}(\mathbb{D})}, \quad 1<p<\infty, \lambda>-1
$$

Gathering all estimates (3.3)-(3.5), we arrive at the conclusion that the Toeplitz operator $T_{a}^{(\lambda)}$ is bounded,

$$
\left\|T_{a}^{(\lambda)} f\right\|_{\mathcal{A}_{\lambda}^{\Phi}(\mathbb{D})} \leqslant C\|f\|_{\mathcal{A}_{\lambda}^{\Phi}(\mathbb{D})}
$$

since the constant $C>0$ can be chosen independently of $f \in \mathcal{A}_{\lambda}^{\Phi}(\mathbb{D})$.

As for the boundedness of the Berezin transform, we have for all $z \in \mathbb{D}$, by the Fubini theorem and the reproducing kernel property,

$$
\begin{aligned}
& \widetilde{T}_{a}^{(\lambda)}(z) \\
= & \int_{\mathbb{D}} \int_{\mathbb{D}} \frac{a(w)\left(1-|w|^{2}\right)^{\lambda}}{(1-\zeta \bar{w})^{2+\lambda}} \frac{\left(1-|z|^{2}\right)^{1+\lambda / 2}}{(1-\bar{z} w)^{2+\lambda}} \frac{\left(1-|z|^{2}\right)^{1+\lambda / 2}}{(1-z \bar{\zeta})^{2+\lambda}}\left(1-|\zeta|^{2}\right)^{\lambda} d A(w) d A(\zeta) \\
= & \int_{\mathbb{D}} \frac{a(w)\left(1-|w|^{2}\right)^{\lambda}}{(1-z \bar{w})^{2+\lambda}} \frac{\left(1-|z|^{2}\right)^{2+\lambda}}{(1-\bar{z} w)^{2+\lambda}} d w=\left(1-|z|^{2}\right)^{1+\lambda / 2} T_{a}^{(\lambda)} k_{z}^{\lambda}(z)
\end{aligned}
$$

The estimate

$$
\left|\frac{\partial^{k}}{\partial w^{k}} k_{z}^{\lambda}(w)\right| \leq C_{k} \frac{\left(1-|z|^{2}\right)^{1+\lambda / 2}}{|1-z \bar{w}|^{2+\lambda+k}}
$$

follows just by differentiating the formula (2.8). For a fixed $z \in \mathbb{D}$, we take $f=k_{z}^{\lambda}$ in (3.3)-(3.4) and apply this to (3.6) so that together with (3.7) we obtain the bound

$$
\begin{aligned}
& \left|\widetilde{T}_{a}^{(\lambda)}(z)\right|=\left(1-|z|^{2}\right)^{1+\lambda / 2}\left|T_{a}^{(\lambda)} k_{z}^{\lambda}(\zeta)\right| \\
\leq & \left(1-|z|^{2}\right)^{1+\lambda / 2} \int_{\mathbb{D}} \sum_{k=0}^{m} \frac{\left(1-|w|^{2}\right)^{k}}{|1-z \bar{w}|^{2+\lambda}}\left|\frac{\partial^{k}}{\partial w^{k}} k_{z}^{\lambda}(w)\right| d A_{\lambda}(w)
\end{aligned}
$$




$$
\begin{aligned}
& \leq\left(1-|z|^{2}\right)^{1+\lambda / 2} \int_{\mathbb{D}} \sum_{k=0}^{m} \frac{\left(1-|w|^{2}\right)^{k}}{|1-z \bar{w}|^{2+\lambda}} \frac{\left(1-|z|^{2}\right)^{1+\lambda / 2}}{|1-z \bar{w}|^{2+\lambda+k}} d A_{\lambda}(w) \\
& =\left(1-|z|^{2}\right)^{2+\lambda} \int_{\mathbb{D}} \sum_{k=0}^{m} \frac{\left(1-|w|^{2}\right)^{k+\lambda}}{|1-z \bar{w}|^{4+2 \lambda+k}} d A(w) .
\end{aligned}
$$

By the Forelli-Rudin-estimates

$$
\int_{\mathbb{D}} \sum_{k=0}^{m} \frac{\left(1-|w|^{2}\right)^{\lambda+k}}{|1-z \bar{w}|^{4+2 \lambda+k}} d A(w) \leq C_{m}\left(1-|z|^{2}\right)^{-2-\lambda}, \quad z \in \mathbb{D},
$$

so that $\left|\widetilde{T}_{a}^{(\lambda)}(z)\right|$ has an upper bound independent of $z$, as claimed.

Theorem 3.4. Let $1<p<\infty$, let $\Phi$ be a Young function, and assume that there exists $m \in \mathbb{N} \cup\{0\}$ such that the average $B_{a, \lambda}^{(m)}$ is nonnegative a.e. in $(0,1)$. If

(ii) the Toeplitz operator $T_{a}^{(\lambda)}: \mathcal{A}_{\lambda}^{\Phi}(\mathbb{D}) \rightarrow \mathcal{A}_{\lambda}^{\Phi}(\mathbb{D})$ is well-defined and bounded, and its Berezin transform $\widetilde{T}_{a}$ is a bounded function,

then we have

$(i) B_{a, \lambda}^{(m+1)}(r)=O\left((1-r)^{m+1+\lambda}\right), \quad r \rightarrow 1$.

Proof. We assume that $T_{a}^{(\lambda)}$ maps $\mathcal{A}_{\lambda}^{\Phi}(\mathbb{D})$ boundedly into itself and the Berezin transform $\widetilde{T}_{a}^{(\lambda)}$ is a bounded function of $z \in \mathbb{D}$. We have by the definition of Berezin transform

$$
\begin{aligned}
\widetilde{T}_{a}^{(\lambda)}(z) & =\widetilde{a}_{\lambda}(z)=\int_{\mathbb{D}} a(w)\left|k_{z}^{\lambda}(w)\right|^{2} d A_{\lambda}(w) \\
& =\frac{\lambda+1}{\pi} \int_{\mathbb{T}}|d \sigma| \int_{0}^{1} a(\rho)\left|k_{z}^{\lambda}(\rho \sigma)\right|^{2}\left(1-\rho^{2}\right)^{\lambda} \rho d \rho \\
& =\frac{\lambda+1}{\pi}\left(1-|z|^{2}\right)^{2+\lambda} \int_{\mathbb{T}}|d \sigma| \int_{0}^{1} a(\rho) \frac{\left(1-\rho^{2}\right)^{\lambda}}{|1-z \rho \bar{\sigma}|^{4+2 \lambda}} \rho d \rho .
\end{aligned}
$$

Here we denoted $w=\rho \sigma$, where $\rho=|w|$ and $\sigma=\frac{w}{|w|}$. If $m=0$, then using Lemma 3.1 we estimate

$$
\begin{aligned}
\left|\widetilde{T}_{a}^{(\lambda)}(z)\right| & =\frac{\lambda+1}{\pi}\left(1-|z|^{2}\right)^{2+\lambda} \int_{\mathbb{T}}|d \sigma| \int_{0}^{1} a(\rho) \frac{\left(1-\rho^{2}\right)^{\lambda}}{|1-z \rho \bar{\sigma}|^{4+2 \lambda}} \rho d \rho \\
& =\frac{\lambda+1}{\pi}\left(1-|z|^{2}\right)^{2+\lambda} \int_{0}^{1} a(\rho)\left(1-\rho^{2}\right)^{\lambda} \rho d \rho \int_{\mathbb{T}} \frac{|d \sigma|}{|1-z \rho \bar{\sigma}|^{4+2 \lambda}}
\end{aligned}
$$




$$
\begin{aligned}
& \geqslant C\left(1-|z|^{2}\right)^{-1-\lambda} \int_{0}^{1} a(\rho)\left(1-\rho^{2}\right)^{\lambda} \rho d \rho \\
& \geqslant C\left(1-|z|^{2}\right)^{-1-\lambda} \int_{|z|}^{1} a(\rho)\left(1-\rho^{2}\right)^{\lambda} \rho d \rho \\
& =C\left(1-|z|^{2}\right)^{-1-\lambda} B_{a, \lambda}^{(1)}(|z|) .
\end{aligned}
$$

This along with the boundedness of the function $\widetilde{T}_{a}^{(\lambda)}$ on $\mathbb{D}$ implies the validity of (i) for $m=0$.

Let now $m \in \mathbb{N}$. Integrating by parts and neglecting the outer terms when $\rho=1$, we have

$$
\begin{aligned}
J_{a, \lambda}(z, \sigma) & \equiv \int_{0}^{1} a(\rho)\left|k_{z}^{\lambda}(\rho \sigma)\right|^{2}\left(1-\rho^{2}\right)^{\lambda} \rho d \rho \\
& =\left(1-|z|^{2}\right)^{2+\lambda} B_{a, \lambda}^{(1)}(0)+\int_{0}^{1} B_{a, \lambda}^{(1)}(\rho) \frac{\partial}{\partial \rho}\left|k_{z}^{\lambda}(\rho \sigma)\right|^{2} d \rho \\
& =\left.\sum_{j=1}^{m} B_{a, \lambda}^{(j)}(0)\left(\frac{\partial^{j-1}}{\partial \rho^{j-1}}\left|k_{z}^{\lambda}(\rho \sigma)\right|^{2}\right)\right|_{\rho=0}+\int_{0}^{1} B_{a, \lambda}^{(m)}(\rho) \frac{\partial^{m}}{\partial \rho^{m}}\left|k_{z}^{\lambda}(\rho \sigma)\right|^{2} d \rho \\
& \equiv J_{a, \lambda}^{(1)}(z)+J_{a, \lambda}^{(2)}(z, \sigma)
\end{aligned}
$$

The term

$$
J_{a, \lambda}^{(1)}(z)=\left.\left(1-|z|^{2}\right)^{2+\lambda} \sum_{j=1}^{m} B_{a, \lambda}^{(j)}(0)\left(\frac{\partial^{j-1}}{\partial \rho^{j-1}} \frac{1}{|1-z \rho \bar{\sigma}|^{4+2 \lambda}}\right)\right|_{\rho=0}
$$

is bounded for each $z \in \mathbb{D}$, and when $|z| \rightarrow 1$ it behaves as

$$
J_{a, \lambda}^{(1)}(z)=O\left(\left(1-|z|^{2}\right)^{2+\lambda}\right), \quad|z| \rightarrow 1 .
$$

Further,

$$
\begin{aligned}
\int_{\mathbb{T}} J_{a, \lambda}^{(2)}(z, \sigma)|d \sigma| & =\int_{\mathbb{T}}|d \sigma| \int_{0}^{1} B_{a, \lambda}^{(m)}(\rho) \frac{\partial^{m}}{\partial \rho^{m}}\left|k_{z}^{\lambda}(\rho \sigma)\right|^{2} d \rho \\
& =\int_{0}^{1} B_{a, \lambda}^{(m)}(\rho) d \rho \frac{\partial^{m}}{\partial \rho^{m}} \int_{\mathbb{T}}\left|k_{z}^{\lambda}(\rho \sigma)\right|^{2}|d \sigma| \\
& =\left(1-|z|^{2}\right)^{2+\lambda} \int_{0}^{1} B_{a, \lambda}^{(m)}(\rho) d \rho \frac{\partial^{m}}{\partial \rho^{m}} \int_{\mathbb{T}} \frac{1}{|1-z \rho \bar{\sigma}|^{4+2 \lambda}}|d \sigma|
\end{aligned}
$$


Hence,

$$
\begin{aligned}
\left|\int_{\mathbb{T}} J_{a, \lambda}^{(2)}(z, \sigma)\right| d \sigma|| & \geqslant C\left(1-|z|^{2}\right)^{2+\lambda} \int_{0}^{1} B_{a, \lambda}^{(m)}(\rho) \frac{1}{(1-\rho|z|)^{3+2 \lambda+m}} d \rho \\
& \geqslant C\left(1-|z|^{2}\right)^{2+\lambda} \int_{|z|}^{1} B_{a, \lambda}^{(m)}(\rho) \frac{1}{(1-\rho|z|)^{3+2 \lambda+m}} d \rho \\
& \geqslant C\left(1-|z|^{2}\right)^{-m-1-\lambda} \int_{|z|}^{1} B_{a, \lambda}^{(m)}(\rho) d \rho \\
& =C\left(1-|z|^{2}\right)^{-m-1-\lambda} B_{a, \lambda}^{(m+1)}(|z|) .
\end{aligned}
$$

Therefore, as above, we see that the condition $(i)$ must be satisfied. This concludes the proof of the necessity of the condition $(i)$.

We reformulate the previous results as the following necessary and sufficient condition concerning the boundedness of the Toeplitz operator. Of course, condition $(i i)$ can be simplified in the (many) cases, where one can deduce the boundedness of the Berezin transform from the boundedness of the Toeplitz operator; see also Remark 2.5.

Theorem 3.5. Let $\Phi$ be a Young function with $1<p_{\Phi} \leqslant q_{\Phi}<\infty$ and assume that there exists $m \in \mathbb{N} \cup\{0\}$ such that the average $B_{a, \lambda}^{(m)}$ is nonnegative a.e. in $(0,1)$. Then, the following are equivalent:

(i) $B_{a, \lambda}^{(m+1)}(r)=O\left((1-r)^{m+1+\lambda}\right), \quad r \rightarrow 1$,

(ii) the Toeplitz operator $T_{a}^{(\lambda)}: \mathcal{A}_{\lambda}^{\Phi}(\mathbb{D}) \rightarrow \mathcal{A}_{\lambda}^{\Phi}(\mathbb{D})$ is well-defined and bounded, and its Berezin transform $\widetilde{T}_{a}^{(\lambda)}$ is a bounded function.

As a corollary we reformulate the Theorem 3.5 for the case of the weighted Lebesgue space (i.e., $\Phi(t)=t^{p}$ ).

Theorem 3.6. Let $1<p<\infty$, and there exists $m \in \mathbb{N} \cup\{0\}$ such that the average $B_{a, \lambda}^{(m)}$ is nonnegative a.e. in $(0,1)$. Then, the following are equivalent:

$(i) B_{a, \lambda}^{(m+1)}(r)=O\left((1-r)^{m+1+\lambda}\right), \quad r \rightarrow 1$,

(ii) the Toeplitz operator $T_{a}^{(\lambda)}: \mathcal{A}_{\lambda}^{p}(\mathbb{D}) \rightarrow \mathcal{A}_{\lambda}^{p}(\mathbb{D})$ is well-defined and bounded.

The reader will have no difficulty to reformulate Theorems 3.3 and 3.4 for the case of the weighted Lebesgue space with $\Phi(t)=t^{p}$. 


\section{References}

[1] D. Bekolle, A. Bonami, E. Tchoundja. Atomic decomposition and Weak Factorization for Bergman-Orlicz spaces. arXiv:1805.03754

[2] S.Charpentier. Small Bergman-Orlicz and Hardy-Orlicz spaces, and their composition operators. Math. Z. (2019). https://doi.org/10.1007/s00209019-02240-w

[3] Y. Deng, L. Huang, T. Zhao, D. Zheng: Bergman projection and Bergman spaces, Journal of Operator Theory. 46 (2001), 3-24.

[4] Duren P., Schuster A. Bergman spaces. Vol. 100. Providence (RI): Mathematical Surveys and Monographs; 2004.

[5] S.M. Grudsky, A.N. Karapetyants, N.L. Vasilevski, Toeplitz operators on the unit ball in $\mathbb{C}^{n}$ with radial symbols. J. Operator Theory. 2003;49:325-346.

[6] S.M. Grudsky, A.N. Karapetyants, N.L. Vasilevski, Dynamics of properties of Toeplitz operators with radial symbols. Integral Equations Operator Theory. 2004;50:217-253.

[7] S.M. Grudsky, A.N. Karapetyants, N.L. Vasilevski,Dynamics of properties of Toeplitz operators in the weighted Bergman spaces. Siberian Electronic Mathematical Reports. 2006;3:362-383.

[8] H. Hedenmalm, B. Korenblum, and K. Zhu, Theory of Bergman Spaces, Springer Verlag, New York, 2000.

[9] P. Harjulehto and P.Hästö. Orlicz spaces and Generalized Orlicz spaces. Lecture Notes in Mathematics, vol. 2236, Springer, Cham, 2019.

[10] A.Karapetyants, H. Rafeiro, S.Samko. Boundedness of the Bergman projection and some properties of Bergman type spaces. Complex Analysis and Operator Theory. 1-15 (2018).

[11] A.Karapetyants, S.Samko. On mixed norm Bergman-Orlicz-Morrey spaces. Georgian Mathematical Journal, Volume 25, Issue 2, 271-282. 
[12] A. Karapetyants,S.Samko. On Boundedness of Bergman Projection Operators in Banach Spaces of Holomorphic Functions in Half-Plane and Harmonic Functions in Half-Space. Journal of Mathematical Sciences. J Math Sci. (2017) Volume 226, Issue 4, pp 344-354

[13] M.A. Krasnoselskii, Ya.B. Rutitski. Convex functions and Orlicz spaces. Translated from the first Russian edition. Noordhoff, Groningen (1961)

[14] A. Kufner, O. John, S. Fucik, Function spaces, Leyden, Noordhoff International Publishing. Monographs and Textbooks on Mechanics of Solids and Fluids. Mechanics: Analysis, 1977

[15] W. Lusky, and J. Taskinen, Toeplitz operators on Bergman spaces and Hardy multipliers, 2011. Studia Mathematica. 204, 2, 137-154

[16] L.Maligranda. Indices and interpolation. Dissertationes Mathematicae 234 (1985).

[17] G. McDonald and C. Sundberg, Toeplitz operators on the disc, Indiana Univ. Math. J. 28 (1979), 595-611.

[18] L. Pick, A. Kufner, O. John, S.Fucik. Function Spaces, 1. De Gruyter Series in Nonlinear Analysis and Applications 14 (2013).

[19] M.M.Rao, Z.D.Ren, Theory of Orlicz spaces, Monographs and Textbooks in Pure and Applied Mathematics, 146, Marcel Dekker, 1991.

[20] B.Sehba. Derivatives characterization of Bergman-Orlicz spaces and applications. arXiv: 1610.01954

[21] I. B. Simonenko. Interpolation and extrapolation of linear operators in Orlicz spaces. Mat. Sb. (N.S.), 1964, Volume 63(105), Number 4, Pages 536-553.

[22] J. Taskinen and J. Virtanen, Toeplitz operators on Bergman spaces with locally integrable symbols. Rev. Mat. Iberoam. 2010; 26 (2): 693-706.

[23] E. Tchoundja, and R. Zhao, Weak Factorization and Hankel Forms for Bergman-Orlicz Spaces on the Unit Ball. Integr. Equ. Oper. Theory (2019) 91: 16 . 
[24] N.L.Vasilevski. Commutative Algebras of Toeplitz Operators on the Bergman Space. Vol.185: Operator Theory: Advances and Applications; 2008 .

[25] S.Yamamuro. Exponents of modulared semi-ordered linear spaces. J. Fac. Sci. Hokkaido Univ. Ser. I Math., Volume 12, Number 4 (1953), 211-253.

[26] K. Zhu, Positive Toeplitz operators on weighted Bergman spaces of bounded symmetric domains, J. Operator Theory 20 (1988), no. 2, 329-357.

[27] K. Zhu, Spaces of Holomorphic Functions in the Unit Ball, Springer Verlag, New York, 2004.

[28] K. Zhu, Operator Theory in Function Spaces, AMS Mathematical Surveys and Monographs 138, 2007.

[29] N. Zorboska, Toeplitz operators with $B M O$ symbols and the Berezin transform, Int. J. Math. Sci., no. 46, (2003) 2929-2945. 